\title{
Molecular Monitoring of Chronic Myeloid Leukemia in Chronic Phase (CML-CP)
}

\author{
Mehrdad Payandeh ${ }^{1}$, Mehrnoush Aeinfar ${ }^{2}$, Saba Yari ${ }^{3}, K_{\text {Khirollah Yari }}^{4,5}$, Masoud \\ Sadeghi ${ }^{2}$
}

${ }^{1}$ Department of Hematology and Medical Oncology, Kermanshah University of Medical Sciences, Kermanshah, Iran. ${ }^{2} \mathrm{Ph} . \mathrm{D}$. in Medical Biology Research Center, Kermanshah University of Medical Sciences, Kermanshah, Iran. ${ }^{3}$ Department of Biology, Faculty of Science, University of Maragheh, Maragheh, Iran. ${ }^{4}$ Medical Biology Research Center, Kermanshah University of Medical Sciences, Kermanshah, Iran. ${ }^{5}$ Razi University Incubator, Zagros Bioidea Company, Kermanshah Iran.

\begin{abstract}
Objective: Quantification of the BCR-ABL transcript is recommended to follow-up CML patients that treated by Imatinib mesylate (IM) as a tyrosine kinase inhibitor. BCR-ABL transcripts have been recognized as a molecular marker for response to therapy in CML patients (pts). Monitoring of this marker to be more effective for identifying optimal responses and can help to inform the decision to switch to alternative therapies. Quantitative reverse transcriptase PCR (Q-PCR) of BCR-ABL1 RNA is a critical laboratory technique for accurate and sensitive monitoring of the efficiency of tyrosine kinase inhibitor therapy. The aim of our study was to analyze the molecular response (MR) in Kurdish CML patients who are treated with Imatinib. Materials and Methods: We studied 60 blood samples from CML patients in chronic phase (CP), 36 females and 24 males, under IM treatment and monitored by Q-PCR on 12 months. The median duration of CML was 5 years (range: 1-15). The median duration of IM treatment was 4 years (range: 1-10). Results: 40\% (24 pts), $28.33 \%$ (17 pts) and 15\% (9 pts) and respectively had reached early molecular response (EMR) at 1.0-2.0 log, major molecular response (MMR) at $3.0 \mathrm{log}$ and deep molecular response (DMR) at 4.0-5.0 log and also undetectable BCR-ABL1 levels (CMR) were achieved in $16.67 \%$ (10 pts) at 12 months. Conclusion: We highlighted the possibility to use of Q-PCR as a warning at diagnosis, and may use to identify patients who could benefit from a more scrupulous follow-up.
\end{abstract}

Keywords: Chronic Myeloid Leukemia- Q-PCR- Molecular Response- Imatinib Mesylate

Asian Pac J Cancer Care, 4 (1), 1-5

\section{Introduction}

Chronic myeloid leukemia (CML) is a hematologic stem cells disorder that determined by increased of myeloid cells in the bone marrow, spleen and peripheral blood [1-2]. The majority of patients with CML ( 80\%) is diagnosed during the initial chronic phase $(\mathrm{CP})$ and usually present with constitutional symptoms, left-shifted neutrophilic splenomegaly, and leukocytosis [3-4]. If untreated, CML progresses to an accelerated phase (AP) and ultimately to a blast phase (BP) [3]. A sign of CML is the presence of $t(9 ; 22)$ ( $q 34 ; q 11)$ translocation and the resulting BCR-ABL1 gene rearrangement, which is known as Philadelphia chromosome $(\mathrm{Ph})$ [1-5]. $\mathrm{Ph}$ is found in more than $90 \%$ of CML patients and typically
Submission Date: 09/02/2018 Acceptance Date: 11/06/2018

leads to $\mathrm{p} 210 \mathrm{kDa} B C \mathrm{~B}-\mathrm{ABL}$ as a chimeric fusion protein [5-6]. This fusion protein is an active tyrosine kinase that is the cause of CML disorder [1-6]. Since the advent of BCR-ABL1 tyrosine kinase inhibitors (TKIs), patients with CML have significantly improved overall survival (OS) and lower rates of disease progressions compared with conventional therapies [7-9]. In 2001 Imatinib mesylate (IM) or Gleevec is a first TKI accepted by the US Food and Drug Administration as an anticancer that targets the adenosine triphosphate (ATP) binding site of the kinase domain of $\mathrm{ABL}$ and blocks the kinase activity of BCR-ABL that is an effective treatment for chronic myeloid leukemia (CML) [10-11]. Three levels of response in CML patients are Hematologic, cytogenetic, and molecular [12]. Complete hematological response

\section{Corresponding Author:}

Dr. Saba Yari

Department of Biology, Faculty of Science, University of Maragheh, Maragheh, Iran.

Email: Sabayari95@gmail.com 
(CHR) is defined as a standardization of the peripheral blood counts with normal peripheral blood smear, and normal spleen [13-14]. Complete cytogenetic response (CCyR) is defined as a response to treatment of CML that absence of the Philadelphia chromosome in the marrow metaphases and Molecular response (MR) is defined as measurement of the reduction of BCR-ABL1 fusion transcripts in the quantitative RT-PCR of blood cells, by molecular methods, it is possible to follow the dynamics of minimal residual disease (MRD) over time [12, 14-16]. So quantitative reverse transcriptase polymerase chain reaction $(\mathrm{Q}-\mathrm{PCR})$ is recommended for accurate and sensitive molecular response (MR) follow-up of treated CML patients these results are expressed as a ratio of BCR-ABL on a control gene [17]. The present study was carried out to monitor the therapeutic response to IM in patients with CML in CP by BCR-ABL Q-PCR techniques.

\section{Materials and Methods}

This study was done at the laboratories in Kermanshah, Iran, between September 2014 and December 2017, 60 adult patients with CML aged 21 to 80 years old, undergoing treatment with Imatinib $400 \mathrm{mg} / \mathrm{day}$ (Table1). Identification of CML was determined by the existence of the BCR-ABL transcripts by RT-PCR. All CML patients identified as Chronic Phase that now on treatment were included in the study. The Chronic Phase was well-defined by the presence of $<15 \%$ blasts, $<20 \%$ basophils and $<30 \%$ blasts and also promyelocytes in both bone marrow and peripheral blood; platelet counts of at least $1 \times 10^{5}$ per cubic millimeter [17-19]. In order to measure BCR-ABL1 transcript levels, $3 \mathrm{~mL}$ of peripheral blood sample was collected in ethylenediaminetetraacetic acid (EDTA)-treated microtube. On the same day, the RNA extraction was done by using RNX-Plus kit (Sinaclon, Iran). Extracted RNA was verified by agarose $(1 \%)$ gel electrophoresis and quantity and quality of extracted RNA were analyzed using a Nanodrop spectrophotometer with the measurement of the absorbance at a wavelength of $260 \mathrm{~nm}$ and the ratio of absorbance at wavelengths of 260 and $280 \mathrm{~nm}$ (260/280), respectively for cDNA synthesis. In order to synthesize of cDNA was used two-step RT-PCR kit (Vivantis, Malaysia). One $\mu \mathrm{g}$ RNA was reverse transcribed with $10 \mathrm{U} / \mu \mathrm{L}$ MMLV, in 1x RT buffer, $25 \mathrm{ng} / \mu \mathrm{L}$ random hexamer primer, $25 \mu \mathrm{M}$ dNTP, $0.01 \mathrm{M} \mathrm{DTT}$, and $2 \mathrm{U} / \mu \mathrm{L}$ RNase inhibitor at $75^{\circ} \mathrm{C}$ for two min, $42^{\circ} \mathrm{C}$ for one $\mathrm{hr}$, and $75^{\circ} \mathrm{C}$ for $10 \mathrm{~min}$. with using of allele-specific primers for p190 and p210, as previously designated by van Dongen et al BCR-ABL1 transcripts were detected by PCR [20]. PCR was done in a total volume of $25 \mu \mathrm{L}$ of a reaction mixture comprising $2.5 \mu \mathrm{L}$ of $10 \mathrm{X}$ PCR buffer, $1 \mu \mathrm{L}$ of $50 \mathrm{mM} \mathrm{MgCl} 2,1 \mu \mathrm{L}$ of $10 \mathrm{mM}$ dNTPs, $2 \mu \mathrm{L}$ of $10 \mu \mathrm{M}$ of forward and reverse primers, $18 \mu \mathrm{L}$ of $\mathrm{H}_{2} \mathrm{O}$ and $0.5 \mu \mathrm{L}$ of $5 \mathrm{U} / \mu \mathrm{L}$ Taq DNA polymerase. The program that was considered for thermal cycler including: initial denaturation for $5 \mathrm{~min}$ at $94^{\circ} \mathrm{C}$, followed by 35 cycles of denaturation for 45 seconds at $94^{\circ} \mathrm{C}$, annealing as 50 seconds at $58^{\circ} \mathrm{C}$, extension 40 seconds at $72^{\circ} \mathrm{C}$ and final extension on 7 minutes at $72^{\circ} \mathrm{C}$. The PCR products were electrophoresed on a $2.5 \%$ Agarose gel with gel red. Pretreatment BCR-ABL1 transcript levels were measured by RT-PCR and after the beginning of IM therapy BCR-ABL1 transcript levels were measured at 12 months, the results were calculated as a percentage of the ratio of BCR-ABL/ABL copy numbers. Molecular response to therapy with IM was defined as: Major molecular response (MMR): if their $\mathrm{BCR}-\mathrm{ABL} / \mathrm{ABL}$ ratios showed a reduction to $0.1 \%$ $(\leq 3 \log )$ or $0.005 \%(\leq 4.5 \log )$; deep molecular response (DMR): if their BCR-ABL/ABL ratios showed $>4$ or $4.5 \mathrm{log}$ reduction. Patients achieving a DMR are possible candidates for treatment free remission trials; complete molecular response (CMR): according to the International Scale (IS), BCR-ABL transcripts not detectable, from a standardized baseline [21]. Also according to the definitions of failure of and suboptimal response to Imatinib suggested by the European Leukemia Network $(\mathrm{ELN})$, these responses were calculated at 12 months (Table 1) [22].

\section{Statistical analyses}

At diagnosis CML, the Sokal index and Hasford risk score were calculated [23-24]. All of the statistical analyses were performed using Excel and R software. A database was created in MS Excel Statistical significance was considered at the $\mathrm{p}<0.05$ level.

\section{Results}

The available demographic and clinical parameters of the CML patients are presented in Table 2. Overall, 60 CML patients with chronic phase were included in our study. There were $24(40 \%)$ male and $36(60 \%)$ female patients. The median duration of CML was 5 years (range: 1 year-15 year) and the median duration of IM treatment was 4 years (range: 1 year-10 year). Sokal Score

Table 1. Definition of the Response to TKIs as First-line Treatment Recommended by ELN

\begin{tabular}{|c|c|c|c|c|}
\hline & & Hematologic Response & Cytogenetic Response & Molecular Response \\
\hline Failure & $\begin{array}{l}\text { 3months } \\
6 \text { months } \\
12 \text { months }\end{array}$ & Not full & $\begin{array}{l}\text { None }\left(\mathrm{ph}^{+}>95 \%\right) \\
\text { Less than partial }\left(\mathrm{ph}^{+}>35 \%\right) \\
\text { Less than complete }\left(\mathrm{ph}^{+}>0\right)\end{array}$ & $\begin{array}{c}\text { BCR-ABL }>10 \% \\
\text { BCR-ABL }>1 \%\end{array}$ \\
\hline Warning & $\begin{array}{l}\text { 3months } \\
6 \text { months } \\
12 \text { months }\end{array}$ & & $\begin{array}{c}\text { Minor/minimal }\left(\mathrm{ph}^{+} 36-95 \%\right) \\
\text { Partial }\left(\mathrm{ph}^{+} 1-35 \%\right)\end{array}$ & $\begin{array}{l}\text { BCR-ABL }>10 \% \\
\text { BCR-ABL } 1-10 \% \\
\text { BCR-ABL } 0.1-1 \%\end{array}$ \\
\hline
\end{tabular}


Table 2. Characteristics of CML Patient

\begin{tabular}{lc}
\hline Variable & CML patients $(\mathrm{N}=60)$ \\
\hline Median age, (range), years & $41(21-80)$ \\
Sex (female/male) & $60(36 / 24)$ \\
Sokal Score & \\
Low & $52(90.6 \%)$ \\
Intermediate & $4(4.7 \%)$ \\
High & $4(4.7 \%)$ \\
Hasford (Euro) Score & \\
Low & $60(100 \%)$ \\
Intermediate & 0 \\
High & 0 \\
Median CML duration, years & $5(1-15)$ \\
The median duration of IM treatment, (range) & $4(1-10)$ \\
WBC (10/L), median (range) & $82(10-630)$ \\
Hemoglobin (g/L), median (range) & $10.9(6-15.6)$ \\
Platelets (10/L), median (range) & $393(13-1280)$ \\
\hline
\end{tabular}

for 52 patients $(86.6 \%), 4(6.7 \%)$ and $4(6.7 \%)$ were low, intermediate and high, respectively, but Hasford (Euro) Score for all patients was low. All of the 60 patients were positive for BCR-ABL at diagnosis time and BCR-ABL: ABL levels in these patients were above and various at baseline. As shown in Figure 1, in our study after 12 months from Imatinib therapy, BCR-ABL: ABL ratio was monitored at baseline. The rates of MMR and DMR at 12 months were $28.33 \%$ and $13.33 \%$, respectively. Also, CMR levels were achieved in $16.66 \%$ at 12 months (Table 3). Of the 60 patients included in the study, $58.33 \%$ finally achieved optimal response. Of the others, $20(33.33 \%)$ and $5(8.33 \%)$ patients respectively had treatment failure and suboptimal response according to the definitions ELN.

\section{Discussion}

For the purpose of targeted therapy for patients with chronic myeloid leukemia (CML), monitoring is vital for optimizing treatment and affordable results. Molecular monitoring in the CML is a noninvasive method to predict outcome and treatment guide decisions. Since 2001 the Imatinib treatment has been used as a front-line therapy in CML patients [7]. This type of treatment is associated with high rates of MMR although a higher rate of CMR (undetectable BCR-ABL) has been reported in patients getting prolonged Imatinib therapy, significant numbers of patients do not achieve MMR, have to be at

Table 3. Log Reduction of BCR-ABL/ABL Ratio at 12 Months while on $400 \mathrm{mg} /$ day Imatinib $\left(\chi^{2}=3.019 ; \mathrm{P}<0.05\right)$

\begin{tabular}{lcc}
\hline Log reduction BCR-ABL/ABL ratio & Frequency & Percent \\
\hline $1<\log$ reduction $\leq 2$ (EMR) & 24 & $40 \%$ \\
$3 \log$ reduction $(\mathrm{MMR})$ & 17 & $28.33 \%$ \\
$4 \leq \log$ reduction $\leq 5$ (DMR) & 9 & $15 \%$ \\
$\mathrm{CMR}=$ Zero & 10 & $16.67 \%$ \\
Total & 60 & 100 \\
\hline
\end{tabular}

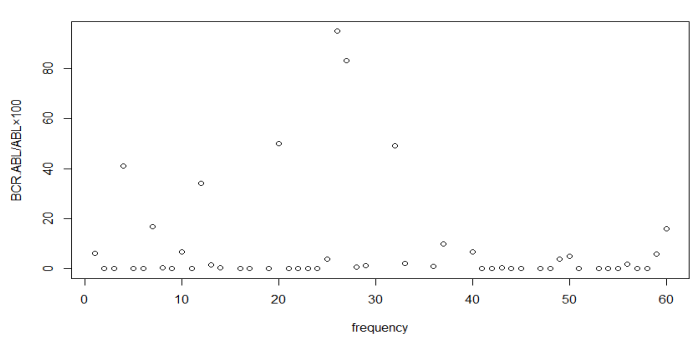

Figure 1. Competitive Quantitative PCR Values at 12 Months on IM Therapy by the Molecular Response

risk for disease progression [25-26]. Increasing levels of BCR-ABL1 RNA indicate the progression of the disease, so the molecular response became an important analytical factor to predict the outcome of the patients treated with TKIs [2]. It is now widely accepted Q-PCR has been used to monitor MRD in CML patients [27].

In our study, the response to treatment is evaluated in patients that received 12 months Imatinib by monitored the BCR-ABL/ABL ratio, and the levels of MMR, DMR, and CMR were $28.33 \%, 15 \%$, and $35 \%$, respectively. These results are relatively comparable with the results reported by Ozatli et al. (2010) that in 77 Turkish CML patients MMR and CMR rates were 58.5\% and 32.5\% at 12 months Imatinib treatment, respectively [28].

But these results are fewer than a Study that performed by Bixby (2009) in 204 subjects were reported 50.1\% MMR at one-year treatment [29].

We supposed that according to different groups of the patients, response rates can be changeable because of gene polymorphisms in different countries and these results might be due to a small sample size. In the future, we hope that will have more samples, so results will be more accurate in representing the population.

The number of patients who achieve a DMR may enable to stop treatment and proper monthly qPCR monitoring [30]. In the case of a contradiction between MR results at prior time points the pathologist should be recommended resampling for validation of results before any decision is taken [25].

suboptimal response and failure treatment, according to the latest ELN recommendations should be considered for a change of treatment [31]. In the present study, 33.33\% of patients didn't the response to $400 \mathrm{mg} /$ day IM so in this case we are faced with two solutions first, change in dose of Imatinib second, a mutation analysis in the kinase domain of BCR-ABL. More than 60 various mutations are identified to be associated with resistance to Imatinib. If Y253H, E255K/V, V299L, T315I, F317L, F359V mutations are identified, the pathologist should indicate which TKI is probably to be more effective against that specific mutation because of resistance is thus associated with a greater risk of disease progression [25].

Though the advent of TKIs has improved patient outcomes, $\mathrm{CML}$ is not easy to manage, but the quantitative determination of residual disease with Q- PCR is a reliable method for monitoring molecular response that provides 
critical analytical data for the management of CML patients which is treated with TKI therapies.

So we conclude that regular molecular monitoring (every 6 or 12 months) of BCR-ABL1 by RT-qPCR provides essential information for evaluating responses as well as for forecasting progression-free survival or relapse during TKI therapy in patients with CML. In this study, $\mathrm{A} \geq 3-\log$ reduction in $75 \%$ patients at 12 months was attained. Patients who achieve $\geq 1-\log$ reduction at 6 months and $\geq 2-\log$ reduction at 12 months are more probably to successively reach MMR [32]. So the early trend in the BCR-ABL/ABL ratio might be clinically useful for the early identification of patients who are destined to achieve an MR on Imatinib.

\section{References}

1. Thompson, P. A., Kantarjian, H. M., \& Cortes, J. E. (2015). Diagnosis and treatment of chronic myeloid leukemia in 2015. In Mayo Clinic Proceedings, 90(10), 1440-1454.

2. Press, R. D., Kamel-Reid, S., \& Ang, D. (2013). BCR-ABL1 RT-qPCR for monitoring the molecular response to tyrosine kinase inhibitors in chronic myeloid leukemia. The Journal of Molecular Diagnostics, 15(5), 565-576.

3. Woessner, D. W., Lim, C. S., \& Deininger, M. W. (2011). Development of an Effective Therapy for CML. Cancer journal (Sudbury, Mass.), 17(6), 477-486.

4. Müller, M. C., Cortes, J. E., Kim, D. W., Druker, B. J., Erben, P., Pasquini, R., ... \& Mukhopadhyay, J. (2009). Dasatinib treatment of chronic-phase chronic myeloid leukemia: analysis of responses according to preexisting BCR-ABL mutations. Blood, 114(24), 4944-4953.

5. Wang, Z., Zen, W., Meng, F., Xin, X., Luo, L., Sun, H., ... \& Huang, L. (2015). Chronic myeloid leukemia with variation of translocation at $(\mathrm{Ph})$ [ins $(22 ; 9)(\mathrm{q} 11 ; \mathrm{q} 21 \mathrm{q} 34)]$ : a case report. International journal of clinical and experimental pathology, 8(10), 13707-13710.

6. Kang, Z. J., Liu, Y. F., Xu, L. Z., Long, Z. J., Huang, D., Yang, Y., ... \& Liu, Q. (2016). The Philadelphia chromosome in leukemogenesis. Chinese journal of cancer, 35(1), 48-62.

7. Kantarjian H, O'Brien S, Jabbour E, Garcia-Manero G, Quintas-Cardama A, Shan J, Rios MB, Ravandi F, Faderl S, Kadia T, et al. (2012). Improved survival in chronic myeloid leukemia since the introduction of imatinib therapy: a singleinstitution historical experience. Blood,119,1981-1987.

8. Björkholm, M., Ohm, L., Eloranta, S., Derolf, Å., Hultcrantz, M., Sjöberg, J., ... \& Kristinsson, S. Y. (2011). Success story of targeted therapy in chronic myeloid leukemia: a population-based study of patients diagnosed in Sweden from 1973 to 2008. Journal of Clinical Oncology, 29(18), 2514-2520.

9. O’brien, S. G., Guilhot, F., Larson, R. A., Gathmann, I., Baccarani, M., Cervantes, F., ... \& Lechner, K. (2003). Imatinib compared with interferon and low-dose cytarabine for newly diagnosed chronic-phase chronic myeloid leukemia. New England Journal of Medicine, 348(11), 994-1004.

10. Hughes, T. P., Kaeda, J., Branford, S., Rudzki, Z., Hochhaus, A., Hensley, M. L., ... \& Radich, J. P. (2003). Frequency of major molecular responses to imatinib or interferon alfa plus cytarabine in newly diagnosed chronic myeloid leukemia. New England Journal of Medicine, 349(15), 1423-1432.

11. Soverini, S., Martinelli, G., Iacobucci, I., \& Baccarani, M. (2008). Imatinib mesylate for the treatment of chronic myeloid leukemia. Expert review of anticancer therapy, 8(6), 853-864.

12. Soverini S, DE Benedittis C, Mancini M, Martinelli G. (2016). Best Practices in chronicmyeloid Leukemia Monitoring and Management. Oncologist, 21, 1-8.

13. Hayran, M., Koca, E., Haznedaroglu, I. C., Unsal, I., Durgun, B., Guvenc, F., ... \& Ozcebe, O. I. (2006). Predicting chronic leukaemias from assessment of complete peripheral blood counts. Journal of international medical research, 34(6), 640-647.

14. Haznedaroglu, I. C. (2014). Monitoring the response to tyrosine kinase inhibitor (TKI) treatment in chronic myeloid leukemia (CML). Mediterranean journal of hematology and infectious diseases, 6(1), 1-7.

15. Baccarani M, Deininger MW, Rosti G, et al. (2013). European LeukemiaNet recommendations for the management of chronic myeloid leukemia: 2013. Blood,122, 872-884.

16. Hughes T, Branford S. (2003). Molecular monitoring of chronic myeloid leukemia. Semin Hematol, 40, 62-68.

17. Marin, D., Kaeda, J., Szydlo, R., Saunders, S., Fleming, A., Howard, J., ... \& Dazzi, F. (2005). Monitoring patients in complete cytogenetic remission after treatment of CML in chronic phase with imatinib: patterns of residual leukaemia and prognostic factors for cytogenetic relapse. Leukemia, 19(4), 507-512.

18. Kantarjian HM, Cortes JE, O’Brien S, Giles F, GarciaManero G, Faderl S, Thomas D, Jeha S, Rios MB, Letvak L, et al. (2003). Imatinib mesylate therapy in newly diagnosed patients with philadelphia chromosome-positive chronic myelogenous leukemia: high incidence of early complete and major cytogenetic responses. Blood, 101(1), 97-100.

19. Cross NC, Hughes TP, Hochhaus A, Goldman JM. (2008). International standardisation of quantitative real-time RTPCR for BCR-ABL. Lukemia Research, 32, 505-506.

20. Van Dongen JJ, Macintyre EA, Gabert JA, Saglio G, Gottardi E, Rambaldi A, Dotti G, Griesinger F, Parreira A, Gameiro P, et al. (1999). Standardized RT-PCR analysis of fusion gene transcripts from chromosome aberrations in acute leukemia for etection of minimal residual disease. Report of the BIOMED-1 concerted action: Investidgation of minimal residual disease in acute leukemia. Leukemia, 13(12), 1901-1928.

21. Baccarani M, Saglio G, Goldman J, Hochhaus A, Simonsson B, Appelbaum F, Apperley J, Cervantes F, Cortes J, Deininger $\mathrm{M}$, et al. (2006). Evolving concepts in the management of chronic myeloid leukemia: recommendations from an expert panel on behalf of the european leukemianet. Blood, 108(6),1809-1820.

22. Baccarani M, Deininger MW, Rosti G, Hochhaus A, Soverini S, Apperley JF, Cervantes F, Clark RE, Cortes JE, Guilhot F, et al. (2013). European leukemianet recommendations for the management of chronic myeloid leukemia: 2013. Blood, 122, 872-874.

23. Hasford, J., Pfirrmann, M., Hehlmann, R., Allan, N. C., Baccarani, M., Kluin-Nelemans, J. C., ... \& Ansari, H. (1998). A new prognostic score for survival of patients with chronic myeloid leukemia treated with interferon alfa Writing Committee for the Collaborative CML Prognostic Factors Project Group. JNCI: Journal of the National Cancer Institute, 90(11), 850-859.

24. Sokal, J. E., Cox, E. B., Baccarani, M., Tura, S., Gomez, G. A., Robertson, J. E., ... \& Cervantes, F. (1984). Prognostic discrimination in" good-risk" chronic granulocytic leukemia. Blood, 63(4), 789-799.

25. Soverini, S., Martinelli, G., Rosti, G., Iacobucci, I., \& Baccarani, M. (2012). Advances in treatment of chronic 
myeloid leukemia with tyrosine kinase inhibitors: the evolving role of $\mathrm{Bcr}-\mathrm{Abl}$ mutations and mutational analysis. Pharmacogenomics, 13(11), 1271-1284.

26. Kantarjian, H., Sawyers, C., Hochhaus, A., Guilhot, F., Schiffer, C., Gambacorti-Passerini, C., ... \& Talpaz, M. (2002). Hematologic and cytogenetic responses to imatinib mesylate in chronic myelogenous leukemia. New England Journal of Medicine, 346(9), 645-652.

27. Yamada, M., Miyamura, K., Fujiwara, T., Yokoyama, H., Tomiya, Y., Ishizawa, K., ... \& Sasaki, T. (2004). Imatinib mesylate in conjunction with allogeneic hematopoietic stem cell transplantation in patients with Philadelphia chromosome positive leukemias: report of 4 cases. The Tohoku journal of experimental medicine, 204(1), 79-84.

28. Ozatli, D., Timuragaoglu, A., Alanoglu, G., Dizlek, S., \& Uysalgil, N. (2010). BCR-ABL transcript level and neutrophil alkaline phosphatase activity in CML patients treated with imatinib. International Journal of Hematology and Oncology, 27(4), 163-168.

29. Bixby, D., \& Talpaz, M. (2009). Imatinib as frontline therapy for patients with newly diagnosed chronic-phase chronic myeloid leukemia. Am Soc Clin Oncol Educ Book, 395-401.

30. Hughes, T. P., \& Ross, D. M. (2016). Moving treatment-free remission into mainstream clinical practice in CML. Blood, 128(1), 17-23.

31. Druker, B. J., Guilhot, F., O’brien, S. G., Gathmann, I., Kantarjian, H., Gattermann, N., ... \& Cervantes, F. (2006). Five-year follow-up of patients receiving imatinib for chronic myeloid leukemia. New England Journal of Medicine, 355(23), 2408-2417.

32. Dewan K, Chatterjee T. (2015). Monitoring of Disease Activity in Chronic Myeloid Leukemia-chronic Phase Patients Treated with Indian Generic Veenat (NATCO) Imatinib Mesylate: A Tertiary Care Experience. Cancer Transl Med,12, 39-42.

\section{c) (i) (우}

This work is licensed under a Creative Commons AttributionNon Commercial 4.0 International License. 